\title{
ABM Clinical Protocol \#23: Non-Pharmacologic Management of Procedure-Related Pain in the Breastfeeding Infant
}

\author{
The Academy of Breastfeeding Medicine Protocol Committee
}

A central goal of The Academy of Breastfeeding Medicine is the development of clinical protocols for managing common medical problems that may impact breastfeeding success. These protocols serve only as guidelines for the care of breastfeeding mothers and infants and do not delineate an exclusive course of treatment or serve as standards of medical care. Variations in treatment may be appropriate according to the needs of an individual patient. These guidelines are not intended to be all-inclusive, but to provide a basic framework for physician education regarding breastfeeding.

\section{Background}

$\mathbf{N}$ EWBORNS AND YOUNG INFANTS routinely experience pain associated with commonly used invasive procedures such as blood sampling and intramuscular injections (e.g., vaccinations, vitamin $\mathrm{K}$ ) and, in some countries, circumcision (the removal of some or all of the foreskin [prepuce] from the penis). ${ }^{1}$ Reduction of pain is both a professional imperative and an ethical expectation because untreated pain has detrimental consequences $^{2}$ such as greater pain sensitivity in later childhood ${ }^{3-6}$ and may lead to permanent neuroanatomical and behavioral abnormalities as demonstrated in animal models. ${ }^{3,7}$ Moreover, pain is a source of concern and distress for new parents and may disturb mother-infant bonding. ${ }^{8}$ Pain reduction therapies are often underused for the numerous minor procedures that are part of routine medical and nursing care for neonates. ${ }^{9,10}$ Growing scientific and clinical evidence from both animal and human newborns points to the efficacy of natural, non-pharmacologic interventions to reduce pain due to minor procedures.

\section{Purpose}

The International Evidence-Based Group for Neonatal Pain and the American Academy of Pediatrics recommend that all neonatal units develop strategies to minimize the number of minor painful or stressful procedures and to provide effective non-pharmacologic and/or pharmacologic pain relief for newborns. ${ }^{11}$ The purpose of this protocol is to provide healthcare professionals with evidence-based guidelines on how to incorporate non-pharmacologic or behavioral interventions to relieve procedure-induced pain in the breastfeeding infant.

\section{Soothing the Newborn}

There are several techniques that have been shown to provide pain relief for newborns (0-28 days of age) undergoing painful procedures. In breastfed newborns, breastfeeding itself is the preferred method to alleviate procedural pain. In addition to being safe, effective, natural, and without added cost, it provides an additional opportunity to promote and support breastfeeding. The individual components of breastfeeding (sucking, sweet taste, and warm contact) may be used separately or, preferably, in combinations when breastfeeding itself is not possible.

\section{Breastfeeding or human milk}

1. When available, breastfeeding should be the first choice to alleviate procedural pain in neonates undergoing a single painful procedure, such as venipuncture or heel lance. $^{12-14}$ Breastfeeding should not be discontinued prior to the procedure. Studies show that when breastfeeding was stopped shortly before a painful procedure, no significant differences were found (compared to control groups) in outcomes in terms of the orogustatory, emotional, tactile, or thermal experience. ${ }^{15}$ When breastfeeding is not possible, whether because of the unavailability of the mother or difficulties with breastfeeding, consider the use of expressed human milk by dropper, syringe, or bottle, which has been shown to soothe newborns experiencing procedural pain. ${ }^{16-19}$ Administration of human milk can also be combined with sucking, by dipping a pacifier (dummy) in the milk, as described below for sucrose.

2. Although some studies have demonstrated the efficacy of human milk alone, ${ }^{17,20}$ human milk may not be equivalent to breastfeeding because of breastfeeding's multicomponent experience. Breastfeeding throughout the painful procedure is likely to be superior to human milk alone on the basis of synergism between the components of breastfeeding. ${ }^{15,20}$

\section{Skin-to-skin contact}

1. Coordinating a breastfeeding session with the timing of the procedure is best, but, if this is not possible, 
skin-to-skin contact can comfort infants undergoing a procedure such as a heel lance. Skin-to-skin contact also gives the mother a caretaking role during the procedure that is unobtrusive, and by diminishing infant stress, it can increase maternal confidence as to her value to the infant. $^{21}$

2. Parental contact and sucrose may act synergistically to reduce pain in neonates. Therefore if feasible, this combination can be employed. ${ }^{22}$ Sucrose taste-first studied 20 years ago - is readily available for increasing the efficacy of other non-pharmacologic techniques. ${ }^{15}$ Sucrose administration is covered in more detail in the section below. Sucrose and pacifier can both be combined with the skin-to-skin component of parental contact.

\section{Sucrose and sucking (in combination or separately)}

Sucrose taste has been shown to be effective analgesia for newborns and young infants for minor procedures, ${ }^{23-25}$ but not for more painful experiences like bladder catherizerations: ${ }^{26}$

1. Sucrose and pacifier. The combination of oral sucrose and pacifier or non-nutritive sucking is remarkably soothing. ${ }^{27}$ This technique offers pain reduction to infants undergoing a wide variety of painful procedures, including heel lance, umbilical or percutaneous venous or arterial catheter insertion, central venous line placement, subcutaneous or intramuscular injection, lumbar puncture, circumcision, and endotracheal suction. ${ }^{1,25,28}$ Because pain reduction achieved when using both sucrose and non-nutritive sucking is similar to that with breastfeeding, using a pacifier (dummy) dipped in $24 \%$ sucrose (by weight) solution whenever breastfeeding is not possible is an effective option. ${ }^{27,29}$ Sucrose administration should begin 2 minutes prior to the procedure. If use of a pacifier is not an available or acceptable option, sucrose can also be combined with sucking by dipping a clean, gloved (or non-gloved parental) finger in the sucrose solution. If sucking a pacifier or finger is not an option, administer a sucrose solution orally before the procedure. ${ }^{1}$ When parents are present, they should be educated that sweet substances other than breastmilk and pacifiers both are recommended in the newborn period only for procedural pain.

2. Glucose versus sucrose. Glucose has also been shown to be an acceptable and effective alternative analgesic. ${ }^{30,31}$ Taste difference is not a factor. Studies in rat $^{32}$ and human $^{33}$ newborns have not shown a preference for sucrose over glucose. The commercial availability of sucrose (table sugar) may have increased its use.

3. Sucrose by syringe. If use of a pacifier is not possible, administer $0.5-2 \mathrm{~mL}$ of a $24 \%$ sucrose solution orally via syringe 2 minutes before the painful procedure. ${ }^{1,34}$ Several $24 \%$ sucrose solutions are commercially available. Sucrose administered by oro- or nasogastric tube is not analgesic.

4. Pacifier alone. While pacifiers alone may decrease crying associated with painful procedures, they do not have the same affect on physiological parameters such as heart rate or vagal tone. ${ }^{35,36}$ Moreover, sucking a paci- fier has been found to reduce pain only when the suck rate exceeds 30 sucks/minute. ${ }^{25}$ A pacifier (or clean gloved or parental finger) should be used as the sole soothing intervention only if breastfeeding, human milk, sucrose (or glucose), and skin-to-skin contact are unavailable because non-nutritive sucking has consistently been found to be better than no intervention at all. ${ }^{37}$

5. Sucrose better than human milk? At least one study indicates that sucrose is more effective than human milk, when both are administered orally via syringe, at reducing infants' cry time, recovery time (heart rate peak returns to baseline), and change in heart rate. ${ }^{23}$ The sugar in human milk is lactose, which has been shown to be an ineffective analgesic agent. ${ }^{32}$ The analgesic component of human milk may be attributed to its fat content or other constituents.

\section{Soothing the Premature Newborn}

Less research has been completed for this population, but there are several techniques that can be used to relieve pain in premature newborns. Breastfeeding may be problematic secondary to the medical status of the infant. Preterm infants may be medically compromised and/or may be developmentally unable to suck or swallow. In such cases, individual components of breastfeeding or a combination of the components of breastfeeding (e.g., contact and sweet taste) are available. Concerns about prolonged sucrose exposure in the premature infant are real. ${ }^{38}$ One study documented infants born at $<31$ weeks who were given a higher number of sucrose doses had lower scores in motor development and attention when assessed at term. ${ }^{39}$ There are no uniform gestational age criteria for studies on analgesia used in preterm infants. The following recommendations are based on studies of infants with an average gestational age of 30 weeks or greater. Not all studies have included infants between 28 and 30 weeks in gestational age, however, and it is unclear if the following recommendations are applicable to that age range. The data do not allow us to extrapolate these recommendations to the smallest premature infants ( $<27$ weeks).

1. Skin-to-skin contact provides effective pain reduction for premature newborns. ${ }^{40,41}$

2. In very-low-birth-weight neonates (27-31 weeks of gestation) undergoing consecutive heel lances, a pacifier dipped in sucrose or in water significantly reduced pain compared with infants who did not receive any intervention. $^{42}$

3. The value of sucrose as a pain reducer in the preterm infant is well established. ${ }^{39,43-45}$ The recommended dosage in this population is $0.1-0.4 \mathrm{~mL}$ of $24 \%$ sucrose solution. ${ }^{1,44}$ Further pain reduction can be achieved when preterm infants receive $24 \%$ sucrose as three doses $(0.1 \mathrm{~mL}, 2$ minutes apart given 2 minutes and immediately prior to heel lance and 2 minutes after lance) rather than as a single dose. ${ }^{45}$

4. The efficacy of breastfeeding and human milk as a pain reducer for the preterm or low-birth-weight infant is not well established in the current literature and should be the subject of further research; no studies have been performed specifically in this population. Regardless, if 
a mother wishes to breastfeed or provide her infant with human milk instead of sucrose, this should not be discouraged.

5. Skin-to-skin contact plus sucrose has not been formally evaluated in premature infants, but may provide pain reduction for the preterm or low-birth-weight neonate.

\section{Soothing the Older Infant (1 Month to 1 Year of Age)}

Breastfeeding or its components as an analgesic technique has not been fully researched across this older population. For children older than a year, the focus of published literature is on the use of distraction techniques, which falls outside the scope of this protocol. ${ }^{46}$

1. Sucrose. Two recent meta-analyses of 10 and 14 randomized clinical trials (RCTs) on infant pain ${ }^{47,48}$ found sucrose to be an effective pain management strategy for infants and children up to 12 months of age. Two milliliters of $25 \%$ sucrose was effective during vaccination up to 6 months of age $;^{49}$ however, $2 \mathrm{~mL}$ of $24 \%$ sucrose was not effective for possibly more painful procedures like bladder catheterization in children older than 1 month of age. ${ }^{26}$ Increasing the concentration of sucrose solution may be more effective as the infant ages. ${ }^{48}$ One study explored the pain-relieving qualities of sucrose in children up to 48 months of age $\mathrm{e}^{50}$ and found effectiveness compared to no treatment. Others, however, report lack of effectiveness with lower concentrations and younger ages. ${ }^{49,51}$ Sucrose taste alone was effective for one vaccination up to 12 months of age $\mathrm{e}^{52}$ but did not demonstrate similar analgesia for multiple (three) vaccinations. ${ }^{53}$ The higher concentrations of sucrose solutions may be more effective at older ages; ${ }^{54}$ however, the majority of studies used differing concentrations, therefore precluding recommendations on the optimal concentration and dose. ${ }^{47,48}$

2. Maternal/caretaker behavior. It has been noted that maternal behavior during a painful procedure accounts for up to $26 \%$ of infant pain behavior during both the procedure and the recovery period. ${ }^{55}$ Maternal distress was an especially important determinant of pain behavior in infants with low vagal tone compared to infants with high vagal tone. ${ }^{56}$ Giving parents a caretaking role, such as securing or distracting the child, can reduce parental sense of helplessness. When parents are unavailable or unable to play a caretaking role, consider recruitment of another person (e.g., nurse) to help secure and/or distract the child. ${ }^{22}$

3. If the infant is still breastfeeding, the mother should be invited to try it. Although the efficacy of breastfeeding and human milk as a pain reducer for older infants has not been extensively studied, there is potential benefit/ minimal risk

4. The upper age limit of effectiveness of sucrose as a pain reducer has not been fully studied, and sucrose therefore cannot be recommended as a pain reducer in infants older than 12 months at this time. ${ }^{22,47,49}$ A recent publication of workshop proceedings reviews the evidence for other techniques such as physical, psychological, and pharmacological interventions and has shown a range of non-pharmacologic treatments to be effective at reducing older childhood vaccine injection pain. ${ }^{47,57-59}$

\section{Recommendations for Future Research}

Further research is needed to establish the most effective non-pharmacologic methods to treat procedural pain for both premature newborns and infants out of the newborn period. In particular, research should focus on the potential of breastfeeding and human milk to reduce pain for premature newborns, newborns experiencing multiple painful procedures, and the older breastfeeding infant. Research is also needed on the effectiveness and effect of increasing concentrations of sweet tastes across different ages in early childhood, as well as the comparison of different combinations of analgesic treatments for older infants/toddlers experiencing procedure-induced pain.

\section{Acknowledgments}

This work was supported in part by a grant from the Maternal and Child Health Bureau, U.S. Department of Health and Human Services.

\section{References}

1. Anand KJS; International Evidence-Based Group for Neonatal Pain. Consensus statement for the prevention and management of pain in the newborn. Arch Pediatr Adolesc Med 2001;155:173-180.

2. Prevention and management of pain and stress in the neonate. American Academy of Pediatrics. Committee on Fetus and Newborn. Committee on Drugs. Section on Anesthesiology. Section on Surgery. Canadian Paediatric Society. Fetus and Newborn Committee. Pediatrics 2000;105:454-461.

3. Ruda MA, Ling QD, Hohmann AG, et al. Altered nociceptive neuronal circuits after neonatal peripheral inflammation. Science 2000;289:628-630.

4. Grunau RE, Oberlander TF, Whitfield MF, et al. Demographic and therapeutic determinants of pain reactivity in very low birth weight neonates at 32 weeks' postconceptional age. Pediatrics 2001;107:105-112.

5. Taddio A, Shah V, Gilbert-MacLeod C, et al. Conditioning and hyperalgesia in newborns exposed to repeated heel lances. JAMA 2002;288:857-861.

6. Oberlander TF, Grunau RE, Whitfield MF, et al. Biobehavioral pain responses in former extremely low birth weight infants at four months' corrected age. Pediatrics 2000; 105(1);e6.

7. Anand KJS, Coskun V, Thrivikraman KV, et al. Long-term behavioral effects of repetitive pain in neonatal rat pups. Physiol Behav 1999;66:627-637.

8. Franck LS, Cox S, Allen A, et al. Parental concern and distress about infant pain. Arch Dis Child Fetal Neonatal Ed 2004;89:F71-F75.

9. Carbajal R, Rousset A, Danan C, et al. Epidemiology and treatment of painful procedures in neonates in intensive care units. JAMA 2008;300:60-70.

10. Simons SHP, van Dijk M, Anand KS, et al. Do we still hurt newborn babies?: A prospective study of procedural pain and analgesia in neonates. Arch Pediatr Adolesc Med 2003; 157:1058-1064.

11. American Academy of Pediatrics Committee on Fetus and Newborn; American Academy of Pediatrics Section on 
Surgery; Canadian Paediatric Society Fetus and Newborn Committee, et al. Prevention and management of pain in the neonate: An update. Pediatrics 2006;118:2231-2241.

12. Codipietro L, Ceccarelli M, Ponzone A. Breastfeeding or oral sucrose solution in term neonates receiving heel lance: A randomized, controlled trial. Pediatrics 2008;122:e716-e721.

13. Carbajal R, Veerapen S, Couderc S, et al. Analgesic effect of breast feeding in term neonates: Randomised controlled trial. BMJ 2003;326:13.

14. Gray L, Miller LW, Philipp BL, et al. Breastfeeding is analgesic in healthy newborns. Pediatrics 2002;109:590-593.

15. Gradin M, Finnström O, Schollin J. Feeding and oral glucose-additive effects on pain reduction in newborns. Early Hum Dev 2004;77:57-65.

16. Mathew PJ, Mathew JL. Assessment and management of pain in infants. J Postgrad Med 2003;79:438-443.

17. Upadhyay A, Aggarwal R, Narayan S, et al. Analgesic effect of expressed breast milk in procedural pain in term neonates: A randomized, placebo-controlled, double-blind trial. Acta Paediatr 2004;93:518-522.

18. Taddio A, Shah V, Hancock R, et al. Effectiveness of sucrose analgesia in newborns undergoing painful medical procedures. CMAJ 2008;179:37-43.

19. Shah PS, Aliwalas LL, Shah VS. Breastfeeding or breast milk for procedural pain in neonates. Cochrane Database Syst Rev 2006;3:CD004950.

20. Shah PS, Aliwalas LL, Shah VS. Breastfeeding or breastmilk to alleviate procedural pain in neonates: A systematic review. Breastfeed Med 2007;2:74-82.

21. Gray L, Watt L, Blass EM. Skin-to-skin contact is analgesic in healthy newborns. Pediatrics 2000;105(1):e14.

22. Schechter NL, Zempsky WT, Cohen PJ, et al. Pain reduction during pediatric immunizations: Evidence-based review and recommendations. Pediatrics 2007;119(5):e1184-e1198.

23. Ors R, Ozek E, Baysoy G, et al. Comparison of sucrose and human milk on pain response in newborns. Eur J Pediatr 1999;158:63-66.

24. Stevens B, Yamada J, Ohlsson A. Sucrose for analgesia in newborn infants undergoing painful procedures. Cochrane Database Syst Rev 2010(1):CD001069.

25. Stevens B, Yamada J, Ohlsson A. Sucrose for analgesia in newborn infants undergoing painful procedures. Cochrane Database Syst Rev 2004;(3):CD001069.

26. Rogers AJ, Greenwald MH, DeGuzman MA, et al. A randomized, controlled trial of sucrose analgesia in infants younger than 90 days of age who require bladder catheterization in the pediatric emergency department. Acad Emerg Med 2006;13:617-622.

27. Blass EM, Watt L. Suckling- and sucrose-induced analgesia in human newborns. Pain 1999;83:611-623.

28. Stevens B. Consistent management of repeated procedural pain with sucrose in preterm neonates: Is it effective and safe for repeated use over time? Clin J Pain 2005;21:543-548.

29. Akman I, Zek E, Bilgen $H$, et al. Sweet solutions and pacifiers for pain relief in newborn infants. J Pain 2002;3:199-202.

30. Axelin A, Salanterä S, Kirjavainen J, et al. Oral glucose and parental holding preferable to opioid in pain management in preterm infants. Clin J Pain 2009;25:138-145.

31. Idam-Siuriun DI, Zhirkova IV, Mikhel'son VA, et al. Prevention of pain during finger prick in neonatal infants [in Russian]. Anesteziol Reanimatol 2008;(1):14-17.

32. Blass EM, Shide DJ. Some comparisons among the calming and pain-relieving effects of sucrose, glucose, fructose and lactose in infant rats. Chem Senses 1994;19:239.
33. Okan F, Coban A, Ince Z, et al. Analgesia in preterm newborns: The comparative effects of sucrose and glucose. Eur J Pediatr 2007;166:1017-1024.

34. Shann F. Suckling and sugar reduce pain in babies. Lancet 2007;369:721-723.

35. Taddio A. Pain management for neonatal circumcision. Paediatr Drugs 2001;3(2):101.

36. Porges SW, Lipsitt LP. Neonatal responsivity to gustatory stimulation: The gustatory-vagal hypothesis. Infant Behav Dev 1993;16:487-494.

37. Pinelli J, Symington A, Siliska D. Nonnutritive sucking in high-risk infants: Benign intervention or legitimate therapy? J Obstet Gynecol Neonatal Nurs 2002;31:582-591.

38. Holsti L, Grunau RE. Considerations for using sucrose to reduce procedural pain in preterm infants. Pediatrics 2010; 125:1042-1047.

39. Johnston CC, Filion F, Snider L, et al. Routine sucrose analgesia during the first week of life in neonates younger than 31 weeks' postconceptional age. Pediatrics 2002;110:523-528.

40. Ludington-Hoe SM, Hosseini R, Torowicz DL. Skin-to-skin contact (Kangaroo Care) analgesia for preterm infant heel stick. AACN Clinical Issues. 2005;16:373-387.

41. Johnston CC, Stevens B, Pinelli J, et al. Kangaroo care is effective in diminishing pain response in preterm neonates. Arch Pediatr Adolesc Med 2003;157:1084-1088.

42. Stevens B, Johnston C, Franck L, et al. The efficacy of developmentally sensitive interventions and sucrose for relieving procedural pain in very low birth weight neonates. Nurs Res 1999;48:35-43.

43. Ramenghi LA, Wood CM, Griffith GC, et al. Reduction of pain response in premature infants using intraoral sucrose. Arch Dis Child Fetal Neonatal Ed 1996;74:F126-F128.

44. Abad F, Díaz NM, Domenech, et al. Oral sweet solution reduces pain-related behaviour in preterm infants. Acta Paediatr 1996;85:854-858.

45. Johnston CC, Stremler R, Horton A, et al. Effect of repeated doses of sucrose during heel stick procedure in preterm neonates. Biol Neonate 1999;75:160-166.

46. Felt BT, Mollen E, Diaz S, et al. Behavioral interventions reduce infant distress at immunization. Arch Pediatr Adolesc Med 2000;154:719-724.

47. Shah V, Taddio A, Rieder MJ. Effectiveness and tolerability of pharmacologic and combined interventions for reducing injection pain during routine childhood immunizations: Systematic review and meta-analyses. Clin Ther 2009; 31(Suppl 2):S104-S105.

48. Harrison D, Stevens B, Bueno M, et al. Efficacy of sweet solutions for analgesia in infants between 1 and 12 months of age: A systematic review. Arch Dis Child 2010;95:406-413.

49. Lewindon PJ, Harkness L, Lewindon N. Randomised controlled trial of sucrose by mouth for the relief of infant crying after immunisation. Arch Dis Child 1998;78:453-456.

50. Dilli D, Küçük IG, Dallar Y. Interventions to reduce pain during vaccination in infancy. J Pediatr 2009;154:385-390.

51. Barr RG, Young SN, Wright JH, et al. "Sucrose analgesia" and diphtheria-tetanus-pertussis immunizations at 2 and 4 months. J Dev Behav Pediatr 1995;16:220-225.

52. Thyr M, Sundholm A, Teeland L, et al. Oral glucose as an analgesic to reduce infant distress following immunization at the age of 3, 5 and 12 months. Acta Paediatr 2007;96: 233-236.

53. Mowery B. Effects of sucrose on immunization injection pain in Hispanic infants. [Ph.D. Thesis]. University of Virginia, Charlottesville, 2007. 
54. Ramenghi LA, Webb AV, Shevlin PM, et al. Intra-oral administration of sweet-tasting substances and infants' crying response to immunization: A randomized, placebo-controlled trial. Biol Neonate 2002;81:163-169.

55. Sweet SD, McGrath PJ. Relative importance of mothers' versus medical staffs' behavior in the prediction of infant immunization pain behavior. J Pediatr Psychol 1998;23:249-256.

56. Sweet SD, McGrath PJ, Dymons D. The roles of child reactivity and parenting context in infant pain response. Pain 1999;80:655-661.

57. Taddio A, Chambers CT, Halperin SA, et al. Inadequate pain management during routine childhood immunizations: The nerve of it. Clin Ther 2009;31(Suppl 2):S152-S167.

58. Taddio A, Ilersich AL, Ipp M, et al. Physical interventions and injection techniques for reducing injection pain during routine childhood immunizations: Systematic review of randomized controlled trials and quasi-randomized controlled trials. Clin Ther 2009;31(Suppl 2):S48-S76.

59. Chambers CT, Taddio A, Uman LS, et al. Psychological interventions for reducing pain and distress during routine childhood immunizations: A systematic review. Clin Ther 2009;31(Suppl 2):S77-S103.
ABM protocols expire 5 years from the date of publication. Evidence-based revisions are made within 5 years or sooner if there are significant changes in the evidence.

Academy of Breastfeeding Medicine Protocol Committee Maya Bunik, M.D., MSPH, FABM ${ }^{*}$ Caroline J. Chantry, M.D., FABM Cynthia R. Howard, M.D., MPH, FABM Ruth A. Lawrence, M.D., FABM

Kathleen A. Marinelli, M.D., FABM, Committee Chairperson Larry Noble, M.D., FABM, Translations Chairperson Nancy G. Powers, M.D., FABM Julie Scott Taylor, M.D., M.Sc., FABM

Contributors *Larry Gray, M.D. *Patel Tanvi, M.D. *Elizabeth Garza, M.D.

*Primary contributors

For correspondence: abm@bfmed.org 
